\title{
Sensitivity Analysis of Fuzzy Activity-Based Life Cycle Costing
}

\author{
Freselam Mulubrhan ${ }^{1, *}$, Ainul Akmar Mokhtar ${ }^{2}$, and Masdi Muhammad ${ }^{2}$ \\ ${ }^{1}$ Universiti Malaysia Pahang, 26300 Gambang, Pahang, Malaysia, \\ ${ }^{2}$ Universiti Teknologi Petronas, 32610 Bandar Seri Iskandar, Perak, Malaysia
}

\begin{abstract}
A sensitivity analysis is typically conducted to identify how sensitive the output is to changes in the input. In this paper, the use of sensitivity analysis in the fuzzy activity based life cycle costing (LCC) is shown. LCC is the most frequently used economic model for decision making that considers all costs in the life of a system or equipment. The sensitivity analysis is done by varying the interest rate and time $15 \%$ and $45 \%$, respectively, to the left and right, and varying $25 \%$ of the maintenance and operation cost. It is found that the operation cost and the interest rate give a high impact on the final output of the LCC. A case study of pumps is used in this study.
\end{abstract}

\section{Introduction}

Life cycle cost (LCC) is one of the supporting tools for decision making. It helps the decision maker to propose and compare different options then select the cost effective options [1]. LCC incorporate all the costs incurred in the life of an equipment or a system. The decision made involves prediction of the future which is filled with potential uncertainties, therefore in order to estimate accurate and efficient LCC the costing method used has a big impact. The costing method used needs to have the ability to grip the uncertainties raised. In this study, Activity Based Costing (ABC) model is used since the model can deal with all the activities associated with costs and uncertainties [2]. In addition to costing method used this uncertain, unclear and incomplete information can be incorporated in the estimation of LCC using soft computing techniques [3]. Fuzzy set theory is one of the soft computing technique which can make the result from the LCC analysis to be inclusive of all the uncertainties [4]. Fuzzy theory is based on the fuzzy sets, which is the expansion of the crisp sets. Fuzzy theory overthrows the two/dual value (i.e. yes or no) so that its multi-value could be pressed close to reality. To identify the key drivers that effect the estimation of the LCC, a sensitivity analysis is applied. A sensitivity analysis is conducted to identify how sensitive the output is to changes in the input and to determine which parameters are the key drivers [5]. Sensitivity analysis can be done either in one way or multiway [6]. If it is needed to analyse the impact of two or more variable varying simultaneously multi-way sensitivity analysis is used. The primary objective of this paper is to determine the most sensitive input of the activity based

${ }^{*}$ Corresponding author : frity $4 \mathrm{u} @$ gmail.com 
LCC using sensitivity analysis. The inputs are acquisition cost, operation cost, maintenance cost, interest rate and service life of the equipment. A case study by Mulubrhan et. al. [7] is used.

\section{Methodology}

In this section, the framework of fuzzy ABC-LCC model is discussed as shown in Fig. 1. The methodology used for this paper is the extension of the methods used by Mulubran et. al. [7]. Mulubran et. al. [7] discusses the methodology for fuzzy activity based costing.

\subsection{Create an activity hierarchy and network}

The first step is forming activity hierarchy; when the hierarchy is made, an activity network is formed. In ABC cost estimating techniques, it is necessary to identify each activity in all the stages, since the principle of activity based costing is that products or services consume activities and these activities consume resources that generate costs. This makes the identified activities to be the cost drivers [7].

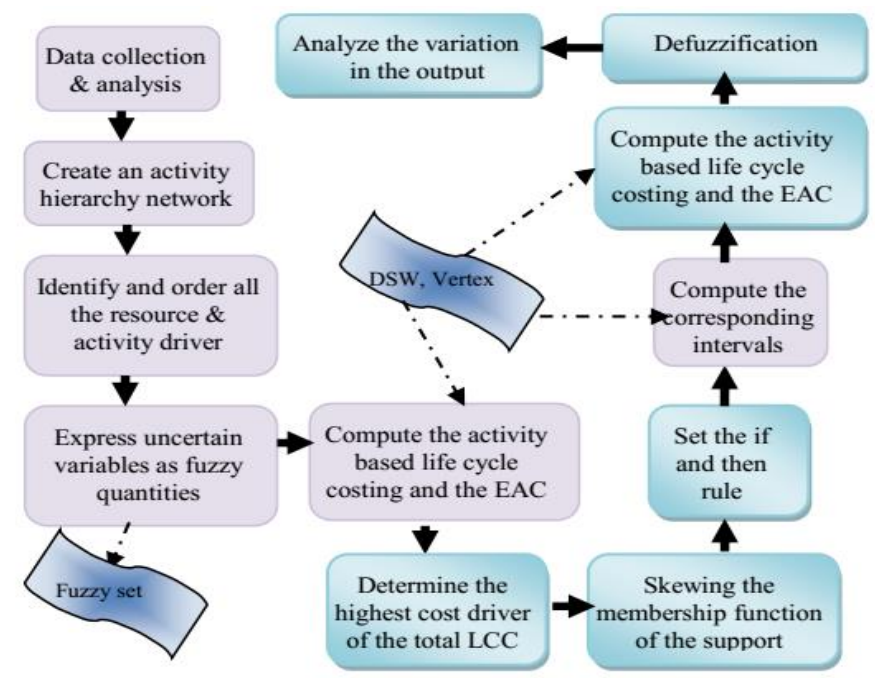

Fig. 1. Schematic representation for framework of fuzzy ABC-LCC model

\subsection{Express the uncertainty variables as fuzzy quantities and select a value for $\alpha$-cut $(0 \leq \alpha \leq 1)$}

Zadeh introduced the fuzzy logic theory where the theory was employed to imitate human thought. It was later found that fuzzy applications were extended to a variety of research areas such as image processing, automated control or even cost analysis. If $X$ is a collection of objects denoted generically by $x$, then a fuzzy set $\tilde{A}$ in $X$ is a set of ordered pairs:

$$
\tilde{A}=\left\{\left(x, \mu_{\tilde{A}}(x)\right) \mid x \in X\right\}
$$

$\mu_{\tilde{A}}$ is called the membership function or grade of membership of $x$ in $\tilde{A}$ that maps $X$ to the membership space $M$ (where $M$ contains only the two points, 0 and 1) [8]. There are different types of fuzzy number such as triangular, trapezoidal, gaussian and sigmoid, to name a few. Triangular fuzzy number is used in widely in many applications as shown in Fig. 2. 


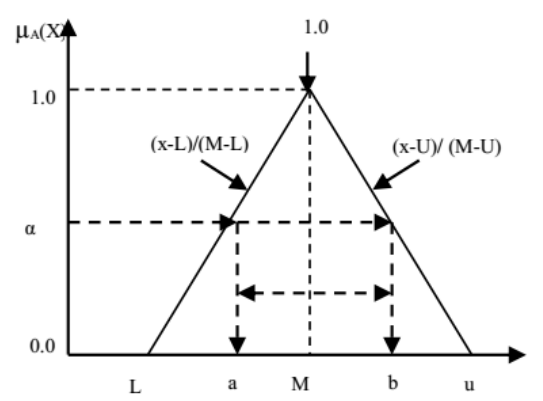

Fig. 2 Triangular membership function with $\alpha$-cut

The membership function, $\mu_{A}(x)$ of a triangular fuzzy number which associated with a real number in the interval $[0,1]$, can be defined as:

$$
\mu_{A}(x)=\left\{\begin{array}{l}
\frac{x}{M-L}-\frac{L}{M-L}, \ldots \ldots \ldots \ldots . . . x \in[L, M] \\
\frac{x}{M-U}-\frac{U}{M-U}, \ldots \ldots \ldots \ldots x \in[M, U]
\end{array}\right.
$$

If $L=M=U$, it becomes ordinary number (non-fuzzy). The sum, subtraction, multiplication and inverse of two triangular fuzzy numbers are similar to crisp numbers. The only different operation is on the inverse which is shown in Eq. (3).

$$
(L, M, U)^{-1} \approx\left(\frac{1}{U}, \frac{1}{M}, \frac{1}{L}\right)
$$

\subsection{Compute the corresponding intervals}

In some complex systems, it would be more reliable to give an interval estimate than a point estimate for many quantities. Interval number $I$ is defined as an ordered pair of real numbers $[a, b]$, with lower bound $a$ and upper bound $b$. When $b=a$, the interval number $[a, a]$ degenerates to a real number $a$ [9]. The $\alpha$-cut of a fuzzy set may also be represented as an interval, such that $A_{\alpha}=[a, b]$, as shown in Fig. 2. The arithmetic operations on any two intervals $[a, b]$ and $[c, d]$ are $[8]$,

$$
\begin{gathered}
\lambda .[a, b]=[\lambda a, \lambda b], \lambda \text { is a constant } \\
{[a, b] \pm[c, d]=[a \pm c, b \pm d]} \\
{[a, b] .[c, d]=[a c, b d]}
\end{gathered}
$$

Dong et al. [6] propose the Day Stout Warren (DSW) algorithm, which is based on the $\alpha$ cut representation of a fuzzy number which is used to choose values for the $\mu(x)$ and computes the corresponding intervals in $X_{1}, X_{2}, \ldots, X_{n}$.

\subsection{Compute the activity-based life cycle costing}

In this section the fuzzy activity based life cycle costing is developed as shown in Eq. (8):

$$
L C C=\tilde{C}_{a q}+\tilde{C}_{o p}+\widetilde{M}_{c}+\widetilde{D}_{c}
$$

where $\tilde{C}_{a q}$ is the acquisition cost, $\tilde{C}_{o p}$ is the operating cost, $\widetilde{M}_{c}$ is the maintenance cost and $\widetilde{D}_{c}$ is the disposal/decomposition cost. All the costs are expressed with a triangular fuzzy number which has the upper value, the modal and the lower value. 


\subsection{Skewing the membership function}

The inputs for the life cycle costing are the acquisition cost, operation cost, maintenance cost, interest rate and time. Each input is stated using one triangular fuzzy number. To determine the effect of the output on the change of the input, the fuzzy number given to the input is skewed to the right and left. That means there are totally three fuzzy numbers to each output.

\subsection{Set the "what if" rules}

As it is stated in the introduction, while one-way sensitivity analysis is useful in demonstrating the impact of one parameter varying in the model, it may be necessary to examine the relationship of two or more different parameters changing simultaneously. To rank the alternative, the fuzzy values have to be converted to a crisp value, for which defuzzification is needed. There are different types of defuzzification method [10]. The most commonly used methods are:

\section{Results and discussions}

A case study of a pump manufactured by the concerned pump manufacturer is chosen to validate the model. The motor used is a low tension (LT) motor, which is easily available in the market. The design life for this pump is 45 years. The data required for the analysis is extracted from [11] [12]. All the activities which are the driver of the cost and their relation can be found in Mulubran et. al. [7]. All costs are summarized in Table 1.

Table 1: Summary of cost for all activities [7]

\begin{tabular}{|c|c|c|c|c|c|c|c|}
\hline Parameter & $\mathbf{L}$ & $\mathbf{M}$ & $\mathbf{U}$ & & & & \\
\hline $\begin{array}{c}\text { Acquisition } \\
\text { Cost }\end{array}$ & 3,647 & 4,330 & 5,076 & $\begin{array}{c}\text { Interest Rate } \\
(\%)\end{array}$ & 4 & 6 & 8 \\
\hline $\begin{array}{c}\text { Operation } \\
\text { Cost }\end{array}$ & 831,650 & $1,039,563$ & $1,247,476$ & $\begin{array}{c}\text { Service Life } \\
\text { (year) }\end{array}$ & 45 & 50 & 55 \\
\hline $\begin{array}{c}\text { Maintenance } \\
\text { Cost }\end{array}$ & 363,766 & 375,000 & 390,220 & & & & \\
\hline
\end{tabular}

In this paper, the $\alpha$-cuts used are $(0,0.2,0.4,0.6,0.8$ and 1$)$. The number of $\alpha$-cuts depends on the function to be calculated and the degree of accuracy needed. The interval for the six $\alpha$-cut values is calculated using DSW algorithm and the present worth factor is calculated using the vertex method for interest rate of and service life. Therefore, the LCC from the given input is found to be $(1651850,1440543,1210005)$. The percentage of all the input costs to the output LCC is found by defuzzification method as shown in Fig. 3. Since the contribution of the acquisition cost is very less, the sensitivity analysis is focused on the operation and the maintenance cost.

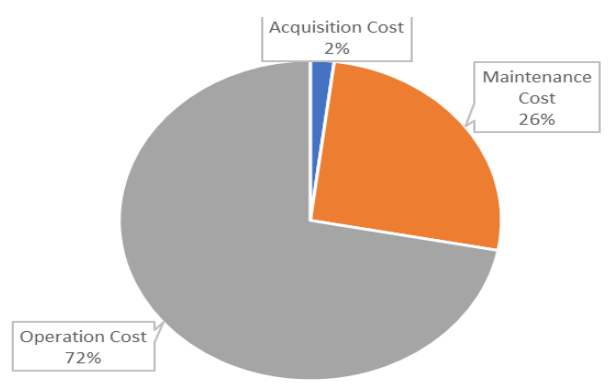


Fig. 3. Percentage of cost inputs to LCC of a pump

Case 1. By making time and interest rate varying $45 \%$ and $15 \%$, respectively, to the left and to the right while the acquisition cost, operation cost, and maintenance cost being fixed. The medium value for time is $(45,50,55)$ and for interest rate, the original value is $(4,6,8)$. These are the input parameters. The input parameters are $(38.25,42.50,46.75)$ for time and $(3.4$, $5.1,6.8$ ) for interest rate when it is shifted $15 \%$ to the left in which it is considered as the lower value. Similarly, the higher value is considered when the inputs are shifted toward right that is $(51.75,57.50,63.25)$ for time and $(4.6,6.9,9.2)$ for interest rate. The triangular fuzzy number of time and interest rate for $45 \%$ and $15 \%$ variation, respectively, is shown in Fig. 4 .
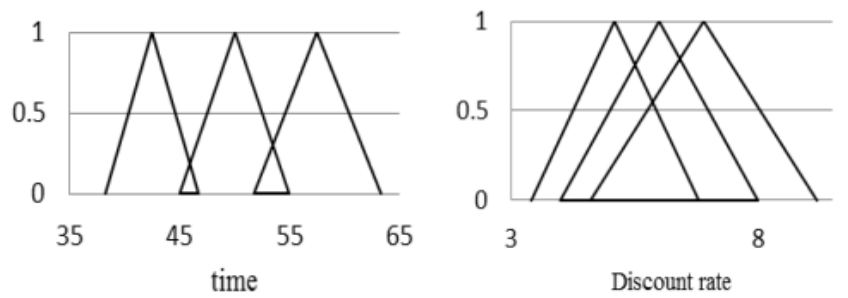

Fig. 4. Triangular fuzzy number of time and interest rate for $45 \%$ and $15 \%$ variation, respectively

The sensitivity of these parameters (i.e. time and interest rate) is checked based on nine combinations of "what if" rules as given below. The analysis is conducted to determine how does the output being affected:

1. If time is low (TL) and interest rate is low (IL)?

2. If time is low (TL) and interest rate is medium (IM)?

3. If time is low (TL) and interest rate is high (IH)?

4. If time is medium (IM) and interest rate is low (IL)?

5. If time is medium (IM) and the interest rate is medium (IM)?

6. If time is medium (IM) and interest rate is high (IH)?

7. If time is high (TH) and interest rate is low (IL)?

8. If time is high (TH) and interest rate is medium (IM)?

9. If time is high (TH) and interest rate is high (IH)?

The result shows that when the time is changed, the output does not have any significant change. However, when the interest rate is changed, the output is changed by $0.27 \%$. Even if the amount is insignificant, it shows that the interest rate is more cost driven compared to the time. To identify the effect of time, the analysis is conducted by varying $45 \%$ for time and $30 \%$ for interest rate. However, the result shows that the interest rate has higher sensitivity and impact factor than the time. The reason for this is that the costs are calculated using equivalent annual cost (EAC) in which maintenance and operation costs are annual cost while the acquisition costs is the present cost. Converting the present cost, which is the acquisition costs, to annual cost does not bring a significant change in the output since the total contribution of acquisition cost is only $2 \%$.

Case 2. By making operation cost and maintenance cost varying $25 \%$ to the left and to the right while acquisition cost, interest rate and time being fixed. The medium value (831650.4, $1039563,1247476)$ for operation cost and $(363766,375000,390220)$ for maintenance cost is the original value of the input parameters. The input parameters are $(623737.8,779672.25$, 935607) for operation cost and $(272824.50,281250,292665)$ for maintenance cost when it is shifted $25 \%$ to the left, in which it is considered as the lower value. Similarly, the higher 
value is considered when the inputs are shifted toward right that is $(1039563,1299454$, $1559345)$ for operation cost and $(454708,468750,487775)$ for maintenance cost. The triangular fuzzy number of operation and maintenance cost is shown in Fig. 5.

(a)

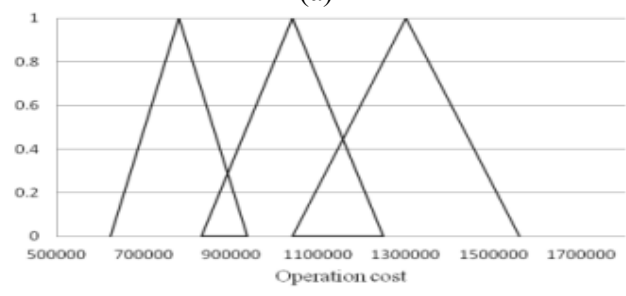

(b)

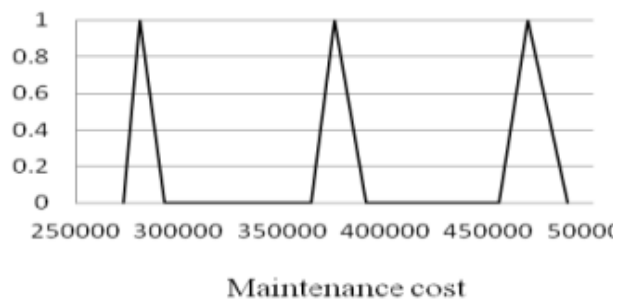

Fig. 5. Triangular fuzzy number of (a) operation and (b) maintenance cost for $25 \%$ variation

The rules for variation of maintenance and operation cost are set likewise interest rate and time. The sensitivity of these parameters is checked based on nine combinations of the "what if" rules as given below. The analysis is conducted to determine how does the output being affected:

1. If operation cost is low (OL) and maintenance cost is low (ML)?

2. If operation cost is low (OL) and maintenance cost is medium (MM)?

3. If operation cost is low (OL) and maintenance cost is high (MH)?

4. If operation cost is medium (OM) and maintenance cost is low (ML)??

5. If operation cost is medium (OM) and the maintenance cost is medium (MM)?

6. If operation cost is medium $(\mathrm{OM})$ and maintenance cost is high $(\mathrm{MH})$ ?

7. If operation cost is high $(\mathrm{OH})$ and maintenance cost is low (ML)?

8. If operation cost is high $(\mathrm{OH})$ and maintenance cost is medium (MM)?

9. If operation cost is high $(\mathrm{OH})$ and maintenance cost is high $(\mathrm{MH})$ ?

The result shows that when the maintenance cost is varied by $25 \%$ to the left and the right while making the other costs constant, the output is changed by $\pm 6.46 \%$. However, the increment and decrement of operation cost is $18.08 \%$ which is three times higher than the maintenance cost. This shows that even if the two parameters increased by the same amount, the operation cost shows high sensitivity by increasing $18 \%$. Fig. 6 shows the increment and decrement of the LCC for variation of maintenance and operation cost.

(a)

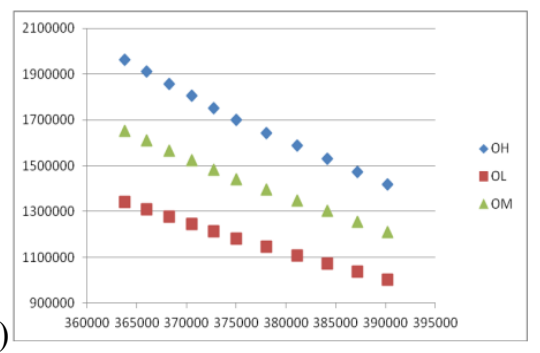

(b)

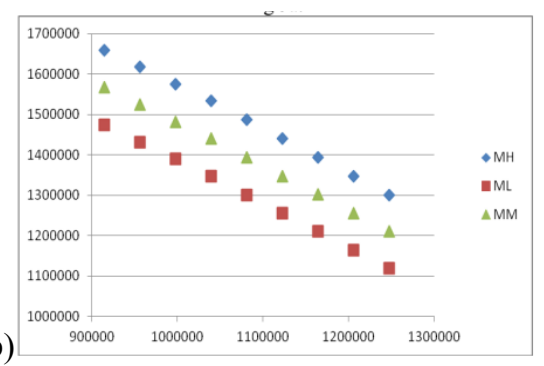

Fig. 6. 25\% change of (a) operation cost and (b) maintenance cost 


\section{Conclusions}

A decision support model is developed by integrating the concept of ABC-LCC, fuzzy logic, interval analysis, DSW and vertex method. Activity based method is used to identify the activities and cost drivers in acquisition, operation and maintenance phase. Fuzzy logic is used to incorporate uncertainties in the cost value. The DSW and the vertex method are helpful in to extending6 ordinary algebraic operations to fuzzy algebraic. Sensitivity analysis is done by varying the interest rate and time $15 \%$ and $45 \%$ to the left and right, and varying maintenance and operation cost $25 \%$ to the left and right. From the analysis it is found that the interest rate and the operation costs are the more sensitive inputs.

\section{References}

1. S. Rahman and D.J. Vanier, CIB 2004 Triennial Congress, 2, 8-11, (2004).

2. J. Emblemsvåg, B. Bras, Activity-Based Cost and Environmental Management. Springer US (2001).

3. C. Chen, G. W. Flintsch, I. L. Al-Qadi. 6th International Conference on Managing Pavements, (2004).

4. E. L. Yu, Master Dissertation, Tunghai University, (1995)

5. W. Dong, H. Shah, Fuzzy Sets System, 24(1), 65-78, (1987)

6. W. M. Dong, H. C. Shah, F. S. Wong, Civil Engineering System, 2(4), 201-208. (1985)

7. F. Mulubran, A. A. Mokhtar, M. Muhammad, International Conference on Mechanical Engineering Research, 74,1-6, (2016).

8. T. Ross, McGraw-Hill, 89-117, (1995)

9. H. J. Zimmermann, Kluwer Academic Publishers,93-111, (2001)

10. A. Chansaad, W. Rattanamanee. Asia Pacific Industrial Engineering \& Management Systems, 1949-1959 (2012)

11. L. Waghmode, A. Sahasrabudhe, P. Kulkarni, y, Journal Mechanical Design, 132(12), 121006-1-1210006-9. [Crossref], [ISI], [Google Scholar] [Infotrieve] ( 2010)

12. F. W. Hennecke, Chemical Engineering and Processing, 38, 511-516, (1999) 